Neurosurg Focus 24 (6):E4, 2008

\title{
Management of bacterial brain abscesses
}

\author{
TaYFun HaKan, M.D. \\ Neurosurgery Clinic, Haydarpaşa Numune Teaching and Research Hospital, Istanbul, Turkey
}

\begin{abstract}
$\checkmark$ Brain abscesses are well-known lesions that have been reported from the beginning of the Hippocratic era. They continue, however, to be characterized by problematic and fatal features, even though there have been enormous developments in treatment and diagnostic technologies - especially in the areas of computed tomography (CT), surgery, anesthesia, bacterial isolation techniques, and new antibiotics. The predisposing factors may change according to patient age, geographic location, and socioeconomic conditions of the community, but patients frequently have a contiguous infection such as otitis or mastoiditis. The clinical signs and symptoms of brain abscesses are nonspecific. Patients typically present with signs and symptoms due to mass effects, accompanied by high fever and seizure. The main treatment is surgical, although medical therapy can be used for selected cases. The treatment of choice is aspiration, which may be performed with the aid of an endoscope or free hand, with or without stereotactic or intraoperative ultrasound guidance. Excision is valuable in some cases. The success of the treatment, whether surgical or medical, mostly depends on the success of isolation of the causative organism, which provides essential data for accurate medical treatment. Third-generation cephalosporins and metronidazole are the most commonly used antimicrobial agents in the treatment of brain abscesses. Use of corticosteroids may be acceptable when lesions are accompanied by edema. Prophylactic antiepileptic therapy is strongly recommended. The patient's Glasgow Coma Scale score at presentation is one of the most important factors predicting outcome. (DOI: 10.3171/FOC/2008/24/6/E4)
\end{abstract}

KEY Words • aspiration • brain abscess • corticosteroid • excision • stereotaxy

Because the newer methods of treatment are good, it does not follow that old ones were bad; for if our honorable and worshipful ancestors had not recovered from their ailments, you and I would not be here today. - Confucius

$\mathrm{B}$ RAIN abscesses are focal suppurative intracranial infections that begin as localized areas of cerebritis in the parenchyma and evolve into collections of pus enclosed by a well-vascularized capsule. The first successful operation for the treatment of a brain abscess, other than surgical treatment performed during the Hippocratic era (460-377 BC), is said to have been performed in 1752, when the French surgeon S. F. Morand ${ }^{5}$ operated successfully on a temporoethmoidal abscess. William Macewen was the first to make a major contribution to the management of brain abscesses; he diagnosed and proposed surgical treatment for a brain abscess in 1876.533 In his classic work, Pyogenic Infectious Disease of the Brain and Spinal Cord. Meningitis, Abscess of the Brain, Infective Sinus Thrombosis, ${ }^{28}$ published in 1893 , he advised draining the abscess and treating the underlying causative sinus infections. His principle that early diagnosis and localization

Abbreviations used in this paper: $\mathrm{CNS}=$ central nervous system; $\mathrm{CT}=$ computed tomography; $\mathrm{MR}=$ magnetic resonance. are the most important factors for treatment of pyogenic brain abscesses was proved with the introduction of CT. ${ }^{5}$ In 1918 , Warrington ${ }^{43}$ investigated the etiological factors in 2 groups: 1) infections from foci in contiguous structures; 2) infections spread through the bloodstream from a distant site. For treatment of the brain abscess, King ${ }^{22}$ introduced marsupialization in 1924 and Dandy introduced aspiration in 1926. Although Sargent ${ }^{37}$ considered the procedure of enucleation of an encapsulated brain abscess in $1928,{ }^{3}$ Vincent ${ }^{42}$ popularized complete excision and proved its value in 1936. In 1971, Heineman and colleagues ${ }^{17}$ became the first to report the successful medical management of a brain abscess.

Even with enormous advances in imaging, surgery, anesthesia, bacterial isolation techniques, and antibiotic therapy, bacterial brain abscesses can still be fatal. ${ }^{19,26}$ The changes in the epidemiology and clinical spectrum of the brain abscess, the predisposing factors, and the prevalence of implicated bacterial pathogens contribute to this mortality in different rates. Brain abscess remains a serious and lifethreatening disease that requires immediate diagnostic and therapeutic attention.

Fundamental to the successful management of bacterial brain abscesses is a multidisciplinary approach; the team should include a neuroradiologist, a neurologist, an infec- 
tious disease specialist, and, of course, a neurosurgeon. In this paper, a comprehensive review of the management of the bacterial brain abscess is presented.

\section{Pathogenesis and Diagnosis}

Causes of brain abscesses may differ according to the socioeconomic conditions of the population and geographic location. Otogenic infection is still one of the most common causative factors in underdeveloped countries. ${ }^{16,19,34}$ The source of infection may be unidentified in $25-38 \%$ of cases, with the resulting brain abscesses classified as "cryptic." ${ }^{26,40,44}$ In the pediatric patient group, the most common predisposing factor is congenital heart disease or adjacent cranial infection..$^{1,2,12,39}$ Recently, Auvichayapat et $\mathrm{al}^{2}$ in their series of 107 cases involving infants and children, found chronic otitis media the second most common predisposing factor after congenital heart disease, and they concluded that this was probably due to the poor socioeconomic status leading to neglect of chronic otitis media, with children going without treatment for years. Brain abscesses in patients with immunosuppression ${ }^{12,40,44}$ and postneurosurgical nosocomial bacterial brain abscesses ${ }^{45}$ are also increasing in incidence.

The clinical symptoms and signs of brain abscesses are nonspecific. Headache, changes in level of consciousness, nausea and/or vomiting, and high fever are the most common manifestations. ${ }^{6,39,40,44}$ Seizure is also not uncommon as an initial symptom, occurring in $25-34 \%$ of patients. ${ }^{2,16} \mathrm{In}$ one case series involving pediatric patients, $27 \%$ of the patients presented with seizure, and none of them had a known history of febrile seizure or epilepsy. ${ }^{12}$

\section{Laboratory Studies}

Such tests as leukocyte count, serum C-reactive protein level, and erythrocyte sedimentation rate are not specific but are valuable especially in the evaluation of the patient's condition during the treatment period. ${ }^{16}$

The microbial investigation of the abscess material is one of the most important factors in the management of a brain abscess. ${ }^{6}$ Gram staining is a simple but important technique that is not to be neglected. It can be positive in cases in which abscess cultures show no microbial growth. ${ }^{19}$ For positive cultures, direct inoculation of the pus specimen immediately after collection from the operation site is important. ${ }^{30}$ The immediate inoculation of the specimen into an anaerobic medium may contribute to better isolation of anaerobic microorganisms. ${ }^{30}$ The most typically isolated microorganisms are Streptococcus and Staphylococcus species. $6,16,19,23,34,40,44$ In a review of 130 cases, Tseng and Tseng ${ }^{40}$ reported that multiple organisms were identified in $23 \%$ of patients. Goodkin et al. ${ }^{12}$ reported that $>1$ organism was isolated in $39 \%$ of 36 positive cultures. Mixed infections that are particularly common in abscesses are those that have an otogenic or mastoid origin. ${ }^{33}$ In their multicenter study, de Louvois et al. ${ }^{9}$ found that cultures were positive for bacteria grown in $100 \%$ of brain abscesses even in the face of antimicrobial treatment. Nevertheless, the rate of negative results for cultures of abscess material can be as high as $34 \% .^{44}$ The empirical use of antibiotics in the preoperative period is assumed to be one of the main causes of the negative culture results. ${ }^{34} \mathrm{In}$ addition to the culture or investigation of pus from the abscess, cultures of blood, ${ }^{6,19}$ sputum, drainage from the ear or sinus, ${ }^{26}$ and cerebrospinal fluid ${ }^{19,26,40,44}$ (if available without a danger of herniation) may be helpful, especially when the results of cultures of the abscess material are negative.

\section{Imaging Studies}

Computed tomography is easily and widely used for confirming the diagnosis and location of the abscesses as well as for follow-up after the treatment period. It is readily available, inexpensive, and fast. ${ }^{19}$ The clinical application of CT opened a new era in the management of brain abscesses. The rate of mortality reduced considerably when compared with the pre-CT period. ${ }^{36}$ Tekkok and Erbengi ${ }^{39}$ found a reduction in mortality rate from $30 \%$ in the era before the use of CT to $6 \%$ in the last 5 years and $0 \%$ in the last 3 years of their study.

Unenhanced CT of an encapsulated abscess reveals a thin ring outlined by the central area of necrosis and peripheral edema; in scans obtained after the administration of a contrast agent, this ring shows strong enhancement. ${ }^{11}$

In addition to CT, MR imaging has contributed greatly to the evaluation of brain abscesses. Findings on both MR images and CT scans vary with the stage of the brain abscess. Mature pyogenic brain abscesses are predominantly hypointense on T1-weighted and hyperintense on T2weighted MR images (Fig. 1). While they display an isointense or slightly hyperintense rim on unenhanced T1weighted images and a hypointense rim on unenhanced T2-weighted images, they show well-defined peripheral rim enhancement on postcontrast T1-weighted images. ${ }^{15,27}$ The cause of the abscess ring on unenhanced images seems to be an accumulation of paramagnetic free radicals. ${ }^{15}$ By using diffusion MR imaging and proton MR spectroscopy it is possible to obtain more sensitive images for discriminating abscesses from cystic tumors or even identifying the nature of the abscess - whether it has a pyogenic, tubercular, or fungal origin. ${ }^{13,27}$

During the treatment period, regular weekly CT examination is advised, followed by monthly CT after discontinuation of antibiotic therapy until the complete resolution of the abscess. ${ }^{33} \mathrm{An}$ immediate CT scan should be done if any deterioration is detected in a patient's status or if no clinical improvement is achieved despite a full course of appropriate therapy (Fig. 2).

\section{Treatment}

The treatment of brain abscesses involves both medical and surgical modalities. The nature of the abscess, its anatomic location, the number of abscesses and their size and stage, as well as the age and initial neurological status of the patient all influence the treatment strategy. ${ }^{26}$ Surgical and medical approaches each have advantages and disadvantages. Medical therapy saves the patient from the stress and complications of the surgery. On the other hand, surgical therapy provides samples for accurate diagnosis, reduces the mass of the abscess, improves the efficacy of the drug used for treatment, and in some conditions allows intrathecal, intraventricular, or intracavitary administration of the antibiotic agent. 

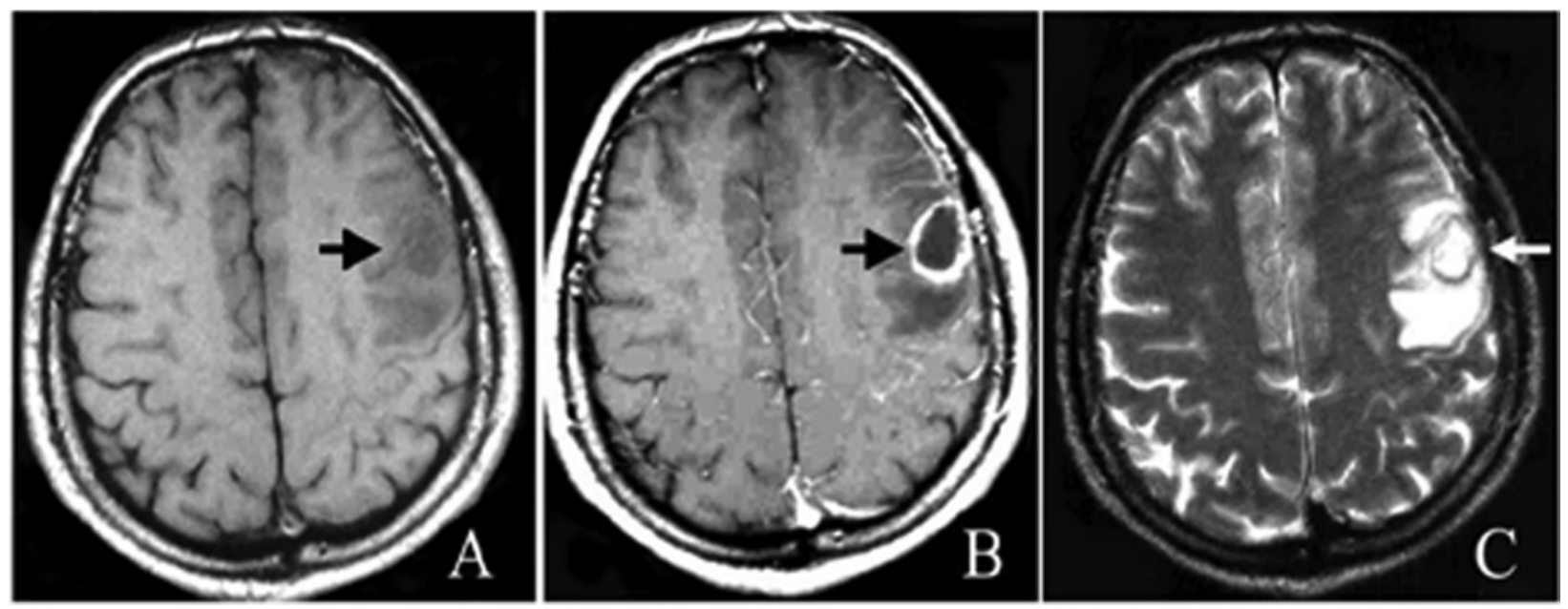

FIG. 1. Axial MR images obtained in a patient with a superficial bacterial abscess (arrows). A: Unenhanced T1weighted image revealing the abscess. B: Contrast-enhanced T1-weighted image showing the abscess with welldefined peripheral rim enhancement. C: T2-weighted image showing the abscess with a hypointense rim and surrounding edema.

\section{Antimicrobial Treatment}

There are few indications for purely nonsurgical treatment of brain abscesses. Medical or nonsurgical treatment alone is indicated for patients with a single abscess smaller than $2 \mathrm{~cm}$, with multiple abscesses, with critical illness at a terminal stage, or with an abscess at an inaccessible localization. ${ }^{6,19,20,40}$ Medical therapy alone may also be considered in abscesses that are in the cerebritis stage of development. ${ }^{20,29}$ The administered antimicrobial agents must be effective against the causative pathogens and capable of passing through the blood-brain barrier in adequate amounts. ${ }^{22}$ Antimicrobial treatment alone is recommended for patients who are in poor systemic condition, ${ }^{22}$ but stereotactic aspiration under local anesthesia can still be very useful in these cases. ${ }^{16}$ Medical treatment is more appropriate and effective in certain patients especially if the agent pathogen is known as a result of a positive culture from cerebrospinal fluid, blood, sputum, or drainage from an ear or the sinuses. ${ }^{26,32}$ When the patient is treated only with medical therapy, the diagnosis should be confirmed by all findings, including clinical signs and symptoms and the results of radiological and microbiological studies. ${ }^{6}$ Medical treatment alone is not recommended when the diagnosis is doubtful and/or confirmation is not available. ${ }^{26}$

When no pathogen has been identified, empirical antimicrobial therapy is used. In such cases, the antibiotic regimen should be selected according to the predisposing condition. With their good CNS penetration and excellent in vitro activity against many of the pathogens that cause brain abscess, such third-generation cephalosporins as cefotaxime or ceftriaxone are used widely., ${ }^{6,184}$ The combination of metronidazole and a third-generation cephalosporin is recommended for the treatment of abscesses originating from otitis, mastoiditis, or sinusitis, and the

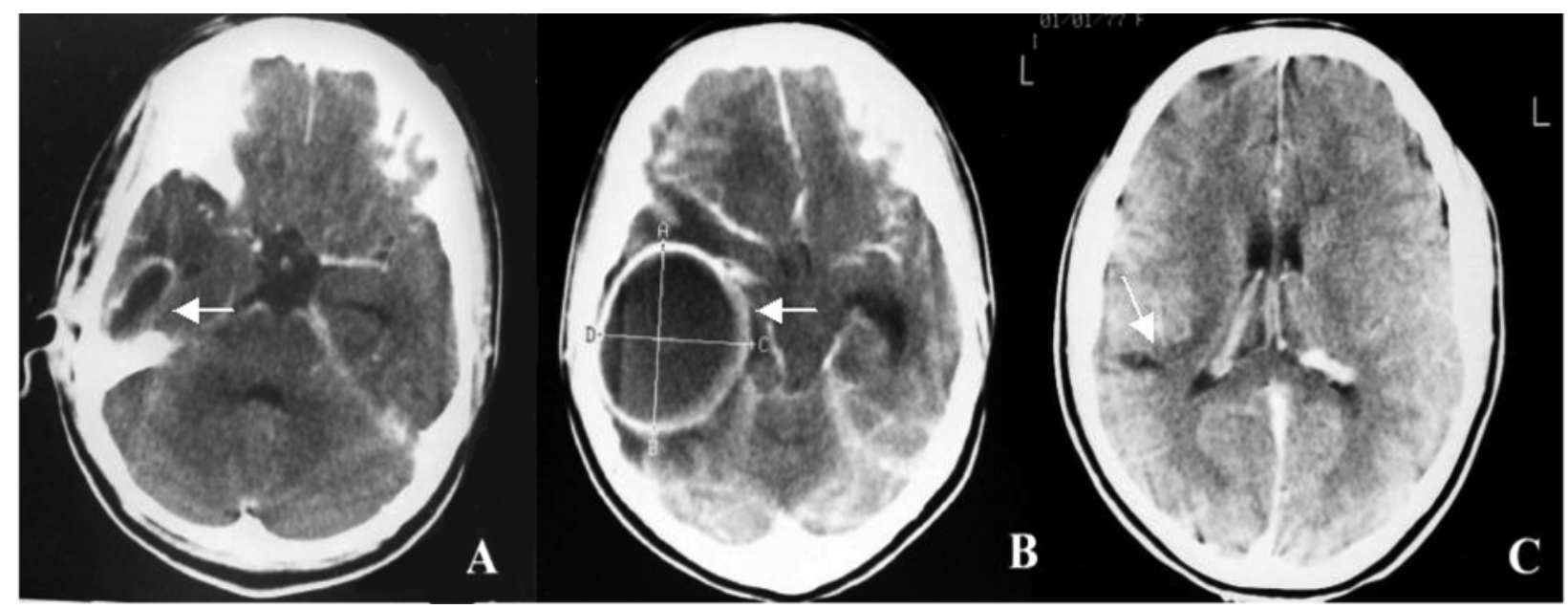

FIG. 2. Axial CT scans obtained in a patient with an otogenic abscess in the right temporal lobe. A: Scan obtained at presentation showing the abscess (arrow) before medical treatment. B: Scan obtained after 7 days of medical treatment showing that the abscess (arrow) has grown and has caused midline shift. C: Image obtained after aspiration, showing a hypoattenuating area (arrow) that remained. The patient recovered with severe sequelae. 
combination of vancomycin and a third-generation cephalosporin is recommended for abscesses associated with trauma or occurring after neurosurgical procedures. ${ }^{41}$ When the source of the infection is obscure, a third-generation cephalosporin can be used in combination with both metronidazole and vancomycin. Depending on the causative pathogen, penicillin $\mathrm{G}$ is recommended mostly for standard therapy of Actinomyces spp., Fusobacterium spp., and Streptococcus spp.; metronidazole for Bacteroides fragilis and third-generation cephalosporins for Enterobacteriaceae and Haemophilus spp. For Staphylococcus spp., the recommended agent is vancomycin, but alternative therapies or modifications always should be kept in mind. ${ }^{41}$ There is no consensus about the duration of antibiotic treatment, but a 6- to 8-week course of parenteral therapy is generally recommended. ${ }^{26,29,45}$

The antibiotics used in the treatment of brain abscesses are expected to reduce CNS bacterial burdens with bactericidal or bacteriostatic properties, to attenuate pathological CNS inflammation that probably contributes to the expansion of the abscess size, to exhibit excellent blood-brain barrier permeability, and to reach high therapeutic levels in the abscess milieu without any unexpected effects. ${ }^{21}$

The patient must be followed up closely clinically and radiologically when a brain abscess is managed medically. ${ }^{33}$ If there is no clinical or radiological improvement despite appropriate medical treatment, surgical treatment should be seriously considered. ${ }^{44}$

\section{Surgical Treatment}

The mainstay of treatment for brain abscesses is a combination of antibiotic treatment and surgical intervention:23 indeed, brain abscess is a surgically treated disease. ${ }^{33}$ Surgery not only obtains pus for accurate bacteriological diagnosis but also decreases the number of pathogens and amount of necrotic tissue present and, most importantly, reduces the mass effect and intracranial pressure. There is a consensus that surgical treatment is indicated for abscesses larger than $2.5 \mathrm{~cm}$ located in noneloquent areas and causing significant mass effect. ${ }^{29,34,40}$ Freehand aspiration, stereotactic aspiration, endoscopic aspiration, and craniotomy with excision are the surgical modalities used for treatment of brain abscesses. ${ }^{14,16,23,25,29,40}$ The choice of treatment modality depends on the patient's status, the techniques available, and the surgeon's experience; there is no significant difference in outcome between aspiration and excision. ${ }^{29,39,40,44}$

Aspiration is the gold standard for treatment of brain abscesses; it is simple and can be easily performed via a bur hole even in critically ill patients at any stage of the abscess. ${ }^{16,26,33}$ In recent series, aspiration is the most often selected method of surgical treatment. $2,6,16,40$ The only contraindication for aspiration is coagulopathy. Moreover, aspiration can be repeated multiple times. ${ }^{9,16}$ In our series, $19 \%$ of the patients treated by means of aspiration underwent the procedure more than once. ${ }^{16}$ Aspiration was performed 4 times in 1 patient, 3 times in 4 patients, and 2 times in the others who underwent repeated aspiration. The use of CT or MR guidance is particularly recommended for aspiration of small or deep-seated abscesses as well as those located in eloquent areas and multiple abscesses. ${ }^{3,16,40}$

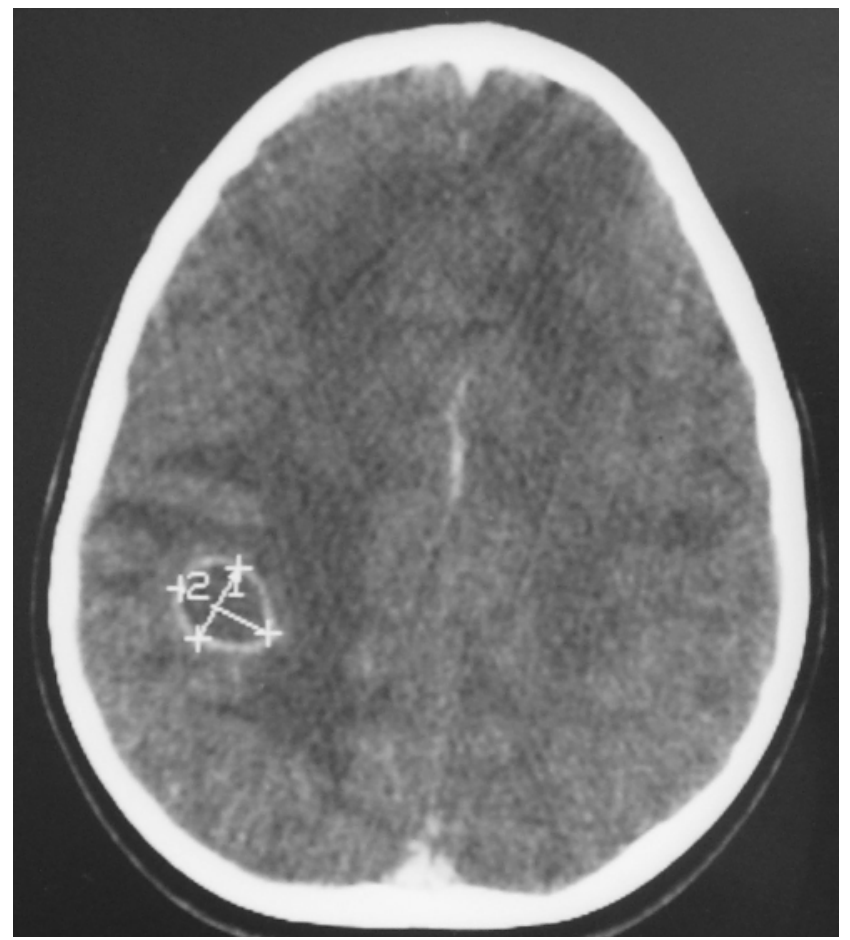

FIG. 3. Axial CT scan obtained in a 6-year-old girl showing a right parietal lobe abscess with ring enhancement $(1.36 \times 1.63$ $\mathrm{cm})$. This image was obtained before the abscess was aspirated and was used for stereotactic guidance during the procedure.

It provides accurate localization, immediate decompression, and in certain cases a biopsy specimen. Abscesses $<$ $3 \mathrm{~cm}$ and $>1.5 \mathrm{~cm}$ in diameter are considered for stereotactic aspiration. ${ }^{32}$ In our study, ${ }^{16}$ we were able to aspirate and obtain a pus sample for bacteriological cultures in an abscess $1.6 \mathrm{~cm}$ in diameter readily and safely (Fig. 3). Intraoperative ultrasound guidance is also very helpful in the surgical treatment of such small abscesses. ${ }^{32,34,44}$ Endoscopic aspiration of brain abscesses is said to be more effective than other aspiration methods; in addition to facilitating retrieval of a specimen and reduction of intracerebral pressure, advantages include direct visual control and the possibility of treating multiseptate abscesses and intraventricular purulent collections..$^{14,25}$

Excision is generally recommended for cerebellar abscesses $^{1,29}$ and abscesses that are superficially located with thick membranes as well as posttraumatic and gas-containing abscesses. ${ }^{7}$ Excision of gas-containing abscesses allows removal of the necrotic materials and closure of possible persistent extracorporal communication. Posttraumatic abscesses frequently contain foreign bodies such as bone fragments that must be removed as well as requiring debridement of necrotic tissue (Fig. 4). ${ }^{33}$ Although excision is advised particularly for multiloculated abscesses, ${ }^{38}$ aspiration of the largest or most reachable part of the lesion for diagnosis and organism identification and treatment with combined antimicrobial therapy that is based on the results of the culture is also a good choice 3 ,29,33 (Fig. 5). Agrawal et al. ${ }^{1}$ report that due to the relatively small volume of the posterior fossa and possible disproportionate edema effect, an abscess in this location may be catastrophic in pediatric 


\section{Bacterial brain abscesses}

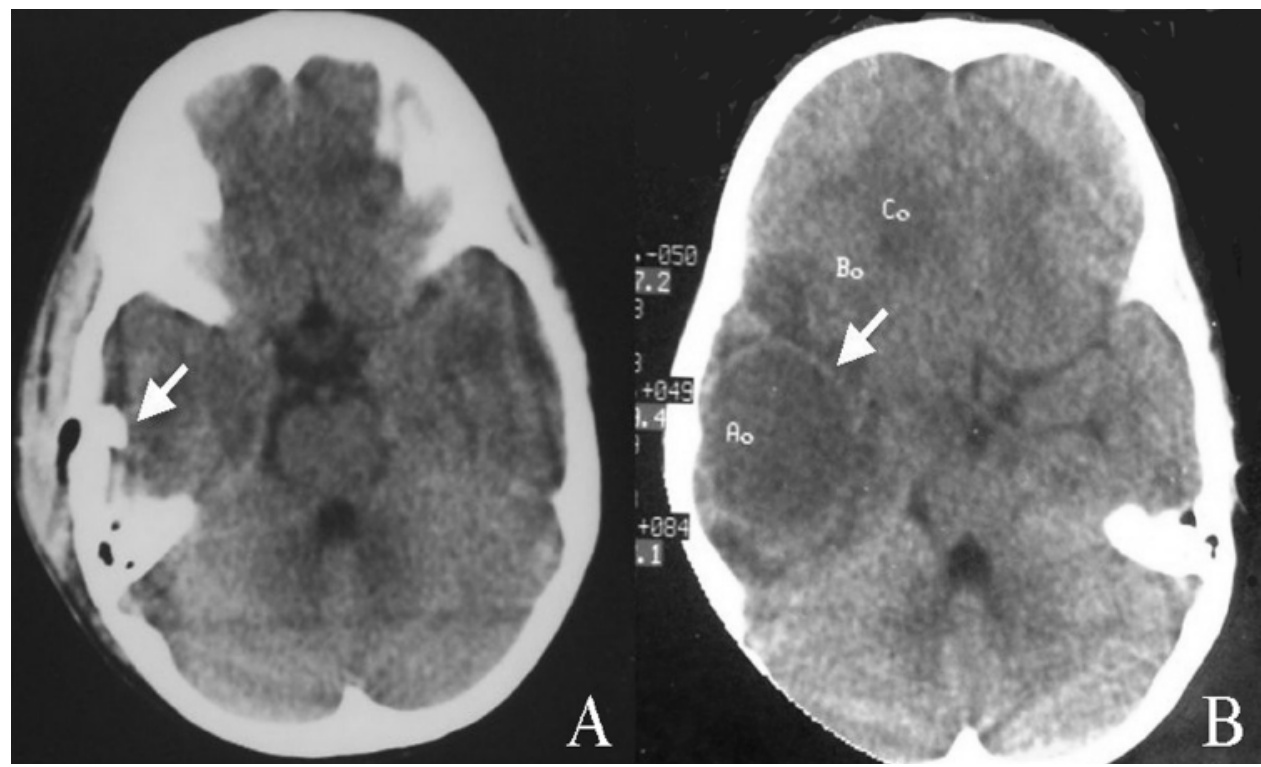

FIG. 4. Axial CT scans obtained in a 4-year-old girl with a traumatic fracture of the right temporal bone. A: Initial scan showing the fracture and a small bone fragment (arrow). The child's parents declined surgical repair. B: Scan obtained 22 days later, after the patient was admitted to the hospital in poor condition, showing that a temporal lobe abscess (arrow) had formed where the bone fragment was seen.

patients; for this reason wide decompression with excision of the abscess is advocated. Abscesses that are resistant to aspiration may also be excised. ${ }^{16}$ In the case series of Agrawal et al., 4 (35\%) of the 14 patients in the excision group had previously been treated with aspiration.

In cases of intraventricular rupture of brain abscesses, in addition to a combination of intrathecal and intravenous antimicrobial treatment, rapid evacuation and debridement of the abscess cavity via urgent craniotomy, lavage of the ventricles, intraventricular drainage, and intraventricular administration of gentamicin are recommended. ${ }^{23,45}$ Intraventricular rupture of the abscess is the most important

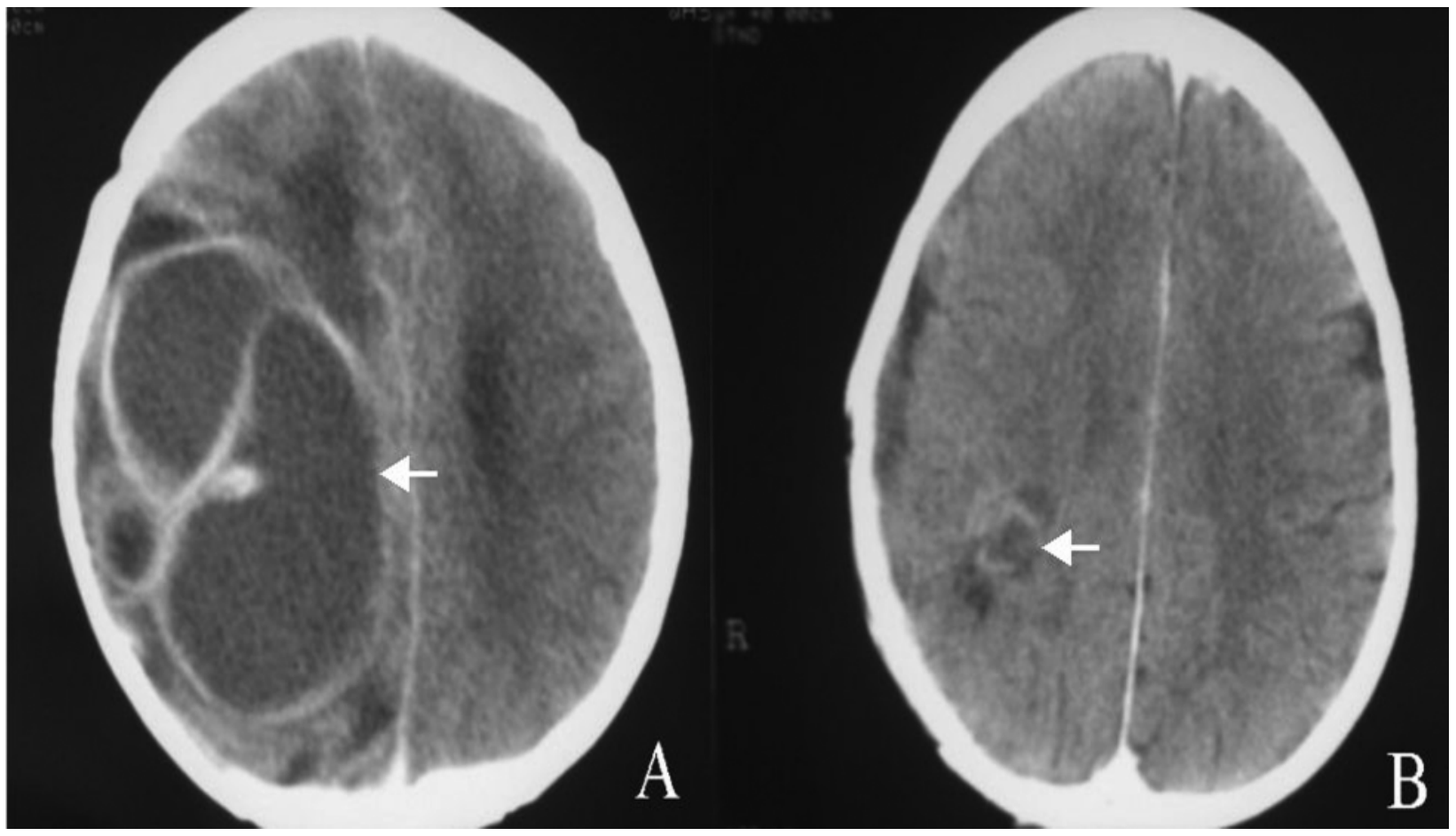

FIG. 5. Axial CT scans obtained in a 2-year-old boy with a right hemisphere abscess. A: Pretreatment image showing an extremely large multiloculated ring-enhancing abscess (arrow). B: Scan obtained after 2 aspirations showing only postoperative changes (arrow) at the site of the abscess. 
complication of brain abscesses; factors associated with increased risk of rupture include deep location, location close to a ventricle wall, and the presence of multiple abscesses. ${ }^{23}$

\section{Corticosteroid Therapy}

The use of corticosteroids in management of brain abscesses is controversial. Local vasogenic edema is the predominant type of edema leading to increased intracranial pressure and significant mortality and morbidity in patients with brain abscesses. ${ }^{4}$ Although there is no well-controlled, randomized clinical study examining the use of corticosteroids for controlling the cerebral edema accompanying brain abscess, corticosteroids are recommended perioperatively for reducing intracranial pressure and avoiding brain herniation. ${ }^{16,19,23,32,39}$ It is important to remember that prolonged use of corticosteroids may decrease the penetration of antimicrobial agents or impair the clearance of some pathogens and may also decrease the enhancement of the abscess wall on radiological examinations, particularly in the cerebritis stage..$^{23,33}$

\section{Anticonvulsive Therapy}

Seizures can occur as one of the initial complications of brain abscesses, and the rates of subsequent attacks are high., ${ }^{216}$ Seizure prophylaxis and continuation of anticonvulsive therapy for an extended period are recommended for patients with brain abscesses. ${ }^{26,29}$

\section{Management of Nonbacterial Abscesses}

Fungal infections of the central nervous system are most often seen in immunocompromised patients. ${ }^{31}$ The frequency with which these infections are identified has been increasing due to the prevalence of acquired immune deficiency syndrome, use of intensive and aggressive chemotherapeutic agents and immunosuppressive regimens, ${ }^{20}$, 24,41 and advances in imaging and microbiological techniques. Yeasts, fungi, and protozoa, including Aspergillus species, Zygomycetes, and Toxoplasma gondii, may also cause brain abscesses with varying incidence. ${ }^{20,35,41}$ Although these infections occur only rarely, they have very poor prognosis and a high mortality rate. Cerebral aspergillosis and mucormycosis, in particular, are very important and dangerous diseases. Cerebral aspergillosis is reported to be present in 10-20\% of all patients with invasive aspergillosis. ${ }^{41}$ Blood vessel invasion leading to thrombosis that causes brain abscess is a characteristic feature of aspergillosis. ${ }^{31}$ The paranasal sinuses and lungs are the most usual sites of infection. ${ }^{24,41}$ Mucormycosis (zygomycosis or phycomycosis) is also a serious, acute, and fulminant fungal infection with a high mortality rate..$^{35,41}$

The treatment modalities used for fungal abscesses are essentially the same as those used for bacterial ones: surgery and medical treatment. The treatment of choice for neuroaspergillosis is surgical excision (debridement) combined with intensive antifungal drug therapy. ${ }^{30,35}$ The combination of amphotericin B and 5-fluorocytosine, which is said to provide synergistic activity, is the recommended antifungal treatment. ${ }^{31,35,41}$

\section{Prognosis}

The major prognostic factors for brain abscesses are early diagnosis, appropriate antimicrobial treatment that is based on causative agents, the virulence of the infecting organisms, and the optimal timing of surgery. ${ }^{19,34,40}$ Demir et al. ${ }^{10}$ reported that number (solitary, multiple), location (superficial, deep, combined), and diameter $(<2 \mathrm{~cm}, 2-4$ $\mathrm{cm},>4 \mathrm{~cm}$ ) of the abscess; and the existence of perilesional edema (minimal, moderate, large) and midline shift $(<5 \mathrm{~mm}, 5-10 \mathrm{~mm},>10 \mathrm{~mm})$ may be used as radiological indicators for prognosis. Most important for early diagnosis is a high index of suspicion. Initial neurological grade, meningismus, high fever $\left(>38^{\circ}\right)$ and leukocytosis $\left(>20,000\right.$ cells per $\mathrm{mm}^{3}$ ) were main factors that were found to influence mortality and sequelae in some recently published papers. ${ }^{16,40}$ Xiao et al. ${ }^{44}$ found that initial Glasgow Coma Scale score, immunodeficiency, and presence of an underlying disease were independently related to outcome.

The mortality rates reported for brain abscesses have varied between $0 \% 1,{ }^{39}$ and $>32 \%$; 19 the mortality rates reported in recent large case series are in the range of $8-25 \%$. $2,6,12,16,34,44$

\section{Conclusions}

Despite advances in diagnosis and treatment, brain abscess remains a life-threatening and important disease. Cases of brain abscess must be evaluated both clinically and radiologically. Abscesses $<2.5 \mathrm{~cm}$ in diameter may be treated with antibiotic therapy alone, but if no improvement is seen (and especially if the patient's condition deteriorates), surgical treatment must be attempted without delay. Aspiration is the gold standard for treatment of brain abscesses. Stereotactic or intraoperative ultrasound guidance may be very useful. The choice of antibiotic agents should be based on culture results when possible; in the absence of positive culture results, therapy with third-generation cephalosporins combined with metronidazole and vancomycin can be considered. Gram staining should not be neglected, as it can provide valuable clues about the infecting organisms. Corticosteroids are used when edema is present, and prophylactic treatment with antiepileptic agents is recommended. The initial clinical status of the patient, high fever, severe leukocytosis, and findings of herniation on radiological examinations are the primary factors influencing the outcome.

Clinicians should have a high index of suspicion for brain abscess because early diagnosis may reduce morbidity and mortality. Infections (including otitis/mastoiditis and sinus, pulmonary, and dental infections) that may lead to brain abscess, particularly in developing countries, should be treated promptly and adequately and the patients should be followed up closely. In all, prevention is cheaper and better for the patients and for the health care community.

\section{References}

1. Agrawal D, Suri A, Mahapatra AK: Primary excision of pediatric posterior fossa abscesses - towards zero mortality? A series of nine cases and review. Pediatr Neurosurg 38:63-67, 2003

2. Auvichayapat N, Auvichayapat P, Aungwarawong S: Brain abscess in infants and children: a retrospective study of 107 patients in northeast Thailand. J Med Assoc Thai 90:1601-1607, 2007

3. Barlas O, Sencer A, Erkan K, Eraksoy H, Sencer S, Bayindir C: 
Stereotactic surgery in the management of brain abscess. Surg Neurol 52:404-411, 1999

4. Bloch O, Papadopoulos MC, Manley GT, Verkman AS: Aquaporin-4 gene deletion in mice increases focal edema associated with staphylococcal brain abscess. J Neurochem 95:254 262, 2005

5. Canale DJ: William Macewen and the treatment of brain abscesses: revisited after one hundred years. J Neurosurg 84:133-142, 1996

6. Carpenter J, Stapleton S, Holliman R: Retrospective analysis of 49 cases of brain abscess and review of the literature. Eur J Clin Microbiol Infect Dis 26:1-11, 2007

7. Colen CB, Rayes M, Rengachary S, Guthikonda M: Outcome of brain abscess by Clostridium perfringens. Neurosurgery 61: E1339, 2007

8. Dandy WE: Treatment of chronic abscesses of the brain by tapping. Preliminary note. JAMA 87:1477-1478, 1926

9. de Louvois J, Gortavai P, Hurley R: Bacteriology of abscesses of the central nervous system: a multicentre prospective study. $\mathbf{B r}$ Med J 2:981-984, 1977

10. Demir MK, Hakan T, Kilicoglu G, Ceran N, Berkman MZ, Erdem I, et al: Bacterial brain abscesses: prognostic value of an imaging severity index. Clin Radiol 62:564-572, 2007

11. Enzmann DR, Britt RH, Placone R: Staging of human brain abscess by computed tomography. Radiology 146:703-708, 1983

12. Goodkin HP, Harper MB, Pomeroy SL: Intracerebral abscess in children: historical trends at Children's Hospital Boston. Pediatrics 113:1765-1770, 2004

13. Fertikh D, Krejza J, Cunqueiro A, Danish S, Alokaili R, Melhem ER: Discrimination of capsular stage brain abscesses from necrotic or cystic neoplasms using diffusion-weighted magnetic resonance imaging. J Neurosurg 106:76-81, 2007

14. Fritsch M, Manwaring KH: Endoscopic treatment of brain abscess in children. Minim Invasive Neurosurg 40:103-106, 1997

15. Haimes AB, Zimmerman RD, Morgello S, Weingarten K, Becker RD, Jennis R, et al: MR imaging of brain abscesses. AJR Am J Roentgenol 152:1073-1085, 1989

16. Hakan T, Ceran N, Erdem I, Berkman MZ, Göktas P: Bacterial brain abscesses: an evaluation of 96 cases. J Infect 52:359-366, 2006

17. Heineman HS, Braude AI, Osterholm JL: Intracranial suppurative disease. Early presumptive diagnosis and successful treatment without surgery. JAMA 218:1542-1547, 1971

18. Jansson AK, Enblad P, Sjölin J: Efficacy and safety of cefotaxime in combination with metronidazole for empirical treatment of brain abscess in clinical practice: a retrospective study of 66 consecutive cases. Eur J Clin Microbiol Infect Dis 23:7-14, 2004

19. Kao PT, Tseng HK, Liu CP, Su SC, Lee CM: Brain abscess: clinical analysis of 53 cases. J Microbiol Immunol Infect 36:129 136, 2003

20. Kastenbauer S, Pfister HW, Wispelwey B, Scheld WM: Brain abscess, in Scheld WM, Whitley RJ, Marra CM (eds): Infections of the Central Nervous System, ed 3. Philadelphia: Lippincott, Williams, \& Wilkins, 2004, pp 479-507

21. Kielian T, Esen N, Liu S, Phulwani NK, Syed MM, Phillips N, et al: Minocycline modulates neuroinflammation independently of its antimicrobial activity in staphylococcus aureus-induced brain abscess. Am J Pathol 171:1199-1214, 2007

22. King JEJ: The treatment of the brain abscess by unroofing and temporary herniation of abscess cavity with avoidance of usual drainage methods, with notes on the management of hernia cerebri general. Surg Gynecol Obstet 39:554-568, 1924

23. Lee TH, Chang WN, Su TM, Chang HW, Lui CC, Ho JT, et al: Clinical features and predictive factors of intraventricular rupture in patients who have bacterial brain abscesses. J Neurol Neurosurg Psychiatry 78:303-309, 2007

24. Lin SJ, Schranz J, Teutsch SM: Aspergillosis case-fatality rate: systematic review of the literature. Clin Infect Dis 32:358-366, 2001
25. Longatti P, Perin A, Ettorre F, Fiorindi A, Baratto V: Endoscopic treatment of brain abscesses. Childs Nerv Syst 22:1447-1450, 2006

26. Lu CH, Chang WN, Lui CC: Strategies for the management of bacterial brain abscess. J Clin Neurosci 13:979-985, 2006

27. Luthra G, Parihar A, Nath K, Jaiswal S, Prasad KN, Husain N, et al: Comparative evaluation of fungal, tubercular, and pyogenic brain abscesses with conventional and diffusion MR imaging and proton MR spectroscopy. AJNR Am J Neuroradiol 28:13321338, 2007

28. Macewen W: Pyogenic Infective Disease of the Brain and Spinal Cord. Meningitis, Abscess of the Brain, Infective Sinus Thrombosis. Glasgow: James Maclehose and Sons, 1893

29. Mamelak AN, Mampalam TJ, Obana WG, Rosenblum ML: Improved management of multiple brain abscesses: a combined surgical and medical approach. Neurosurgery 36:76-86, 1995

30. Marinovic T, Skrlin J, Vilendecic M, Rotim K, Grahovac G: Multiple Aspergillus brain abscesses in immuno-competent patient with severe cranio-facial trauma. Acta Neurochir (Wien) 149: 629-632, 2007

31. Nadkarni T, Goel A: Aspergilloma of the brain: an overview. J Postgrad Med 51 (1 Suppl): S37-S41, 2005

32. Obana WG, Rosenblum ML: Nonoperative treatment of neurosurgical infections. Neurosurg Clin N Am 3:359-373, 1992

33. Osenbach RK, Loftus CM: Diagnosis and management of brain abscess. Neurosurg Clin N Am 3:403-420, 1992

34. Prasad KN, Mishra AM, Gupta D, Husain N, Husain M, Gupta RK: Analysis of microbial etiology and mortality in patients with brain abscess. J Infect 53:221-227, 2006

35. Redmond A, Dancer C, Woods ML: Fungal infections of the central nervous system: a review of fungal pathogens and treatment. Neurol India 55:251-259, 2007

36. Rosenblum ML, Hoff JT, Norman D, Weinstein PR, Pitts L: Decreased mortality from brain abscesses since advent of computerized tomography. J Neurosurg 49:658-668, 1978

37. Sargent P: Remarks on drainage of brain abscess. Br Med J 2: 971-972, 1928

38. Su TM, Lan CM, Tsai YD, Lee TC, Lu CH, Chang WN: Multiloculated pyogenic brain abscess: experience in 25 patients. Neurosurgery 52:1075-1080, 2003

39. Tekkök IH, Erbengi A: Management of brain abscess in children: review of 130 cases over a period of 21 years. Childs Nerv Syst 8:411-466, 1992

40. Tseng JH, Tseng MY: Brain abscess in 142 patients: factors influencing outcome and mortality. Surg Neurol 65:557-562, 2006

41. Tunkel AR: Brain abscess, in Mandell GL, Bennett JE, Dolin R (eds): Principles and Practice of Infectious Disease, ed 6. Philadelphia: Elsevier Churchill Livingston, 2005, Vol 1, pp $1150-1163$

42. Vincent C: Sur une méthode de traitement des abcès subaigus des hémisphères cérébraux: large décompression, puis ablation en masse sans drainage. Gaz Méd de Fr 43:93-96, 1936

43. Warrington WB: Abscess of the brain. Q J Med 2:141-164, 1918

44. Xiao F, Tseng MY, Teng LJ, Tseng HM, Tsai JC: Brain abscess: clinical experience and analysis of prognostic factors. Surg Neurol 63:442-450, 2005

45. Yang KY, Chang WN, Ho JT, Wang HC, Lu CH: Postneurosurgical nosocomial bacterial brain abscess in adults. Infection 34:247-251, 2006

Manuscript submitted February 1, 2008.

Accepted March 3, 2008.

Address correspondence to: Tayfun Hakan, M.D., Neurosurgery Clinic, Haydarpaşa Numune Teaching and Research Hospital, Istanbul, Turkey. email: tayfunhakan@yahoo.com. 